\title{
Experimental study on the forcing design for an intermittent injection
}

\author{
Giovanni Lacagnina ${ }^{1,2}\left([) \cdot\right.$ Robert-Zoltán Szász $^{3}\left([) \cdot\right.$ Lisa Prahl Wittberg $^{2}\left(\right.$ Laszlo Fuchs $^{2}$
}

Received: 22 September 2017 / Revised: 29 May 2018 / Accepted: 1 June 2018 / Published online: 3 July 2018

(c) The Author(s) 2018

\begin{abstract}
In this paper we consider the effects of acceleration and deceleration on the forcing of an intermittent jet. This experimental study specifically focuses on the effect of the acceleration and deceleration on the mixing of an intermittent jet with the ambient fluid and on the growth of disturbances that may lead to turbulence. The influence of different injection strategies has been evaluated. The results show that the deceleration phase may be able to contribute significantly to enhance the mixing of the jet with the ambient fluid. This effect is manifested primarily around the tail of the jet, towards the end of injection. The acceleration phase on the other hand has mainly impact at the leading part of the jet, where the leading part of the jet forms a mushroom shaped structure with minor mixing effect.
\end{abstract}

Giovanni Lacagnina

G.Lacagnina@soton.ac.uk

1 Institute of Sound and Vibration Research (ISVR),

University of Southampton, Southampton, UK

2 Department of Mechanics, KTH Royal Institute of Technology, Stockholm, Sweden

3 Department of Energy Sciences, LTH Faculty of Engineering, Lund University, Lund, Sweden 


\section{Graphical abstract}
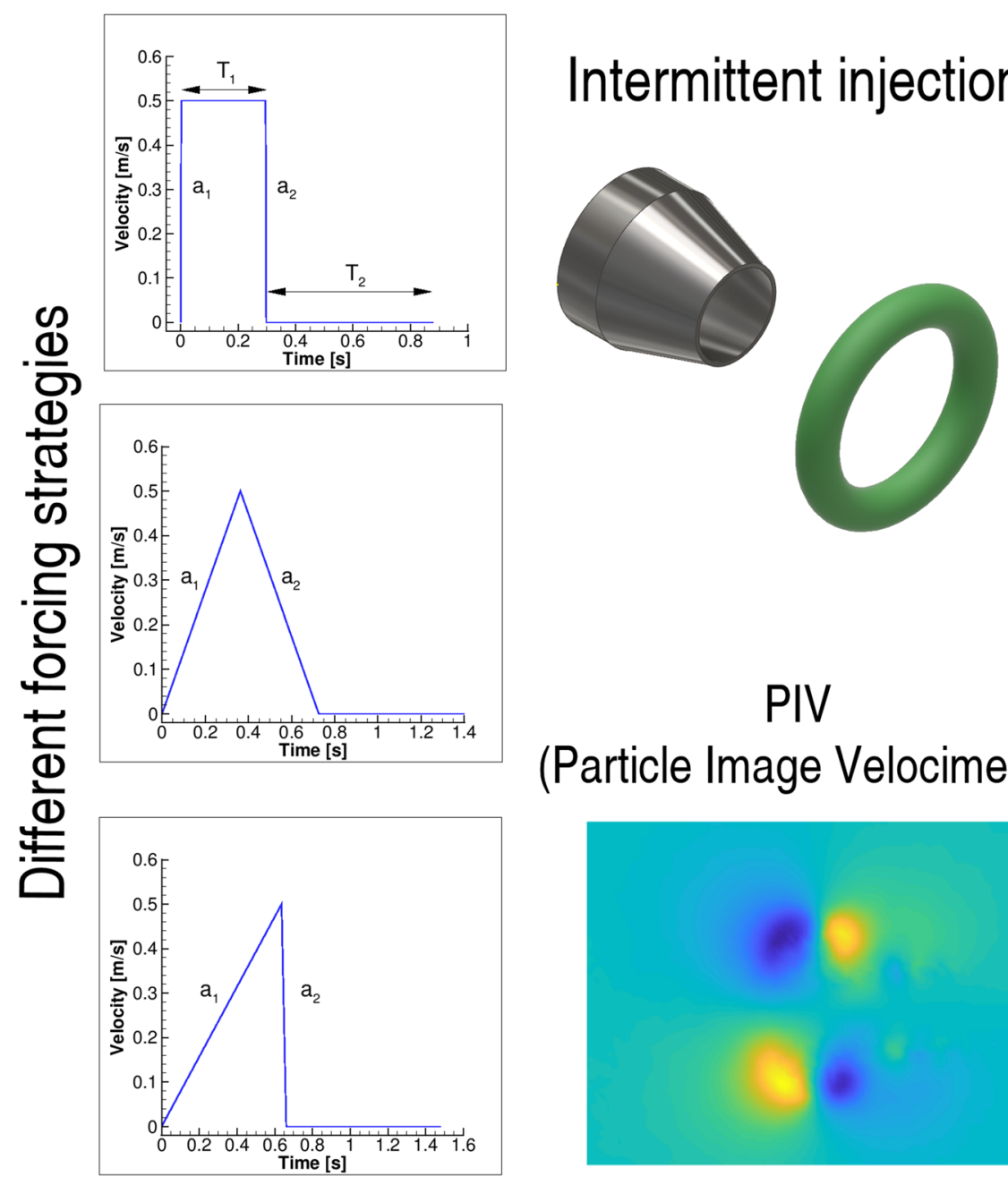

Entrainment rate
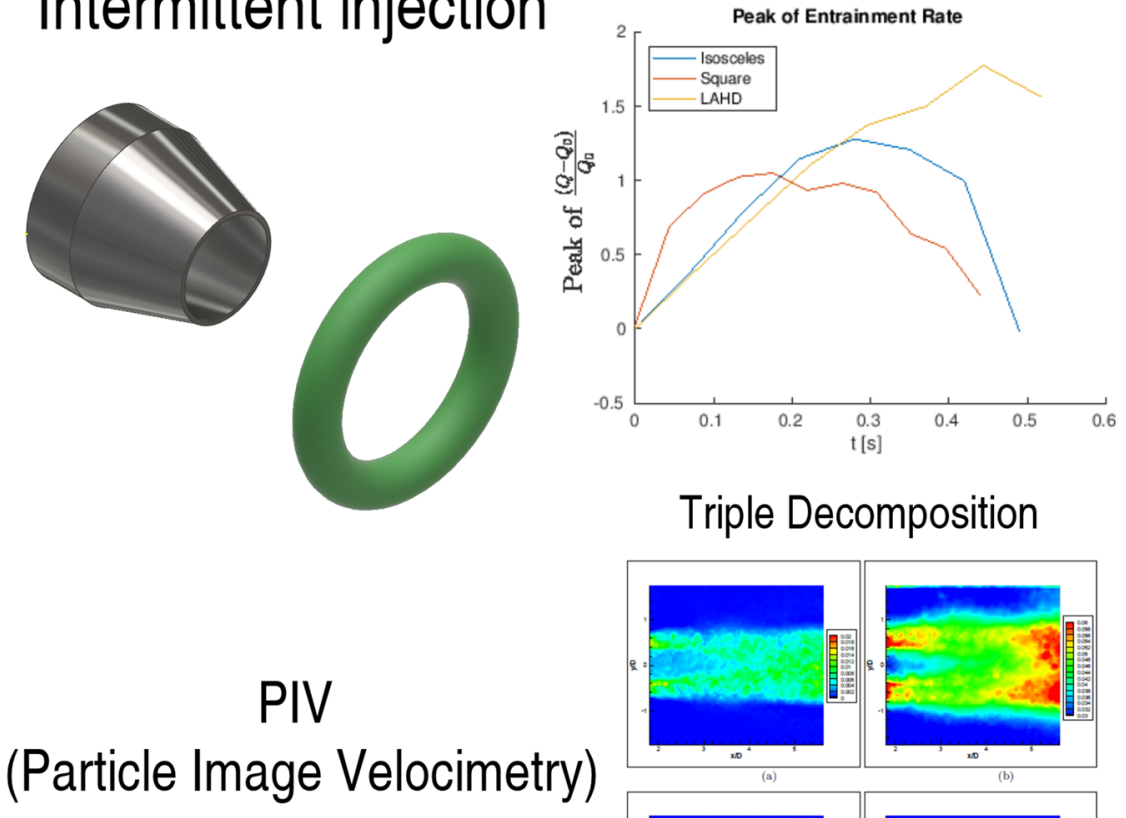

Triple Decomposition
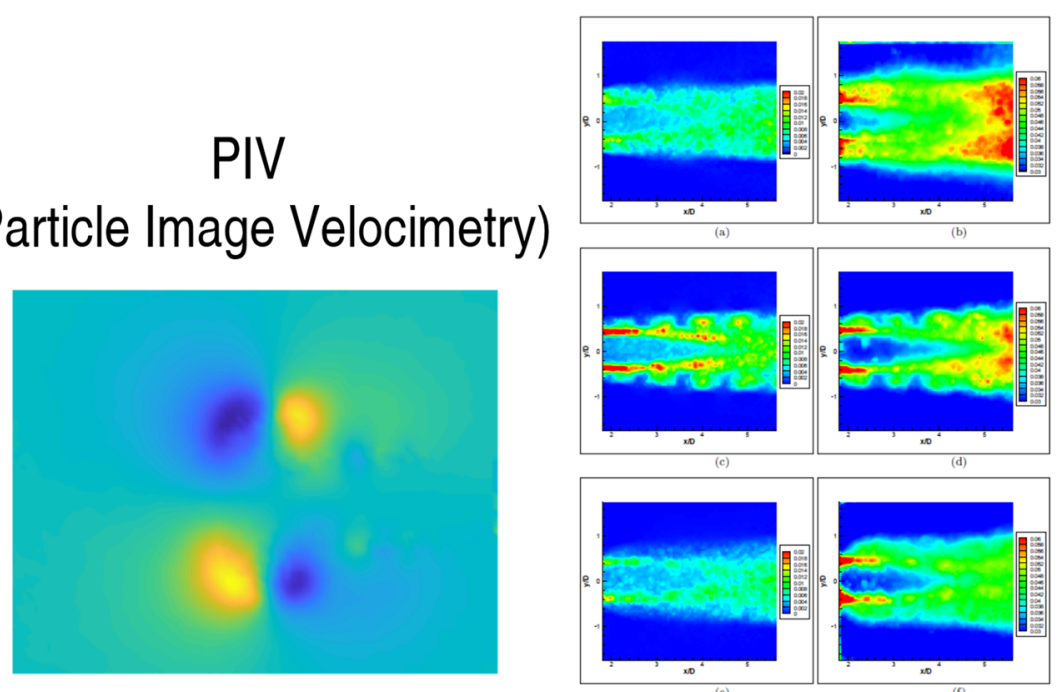

\section{Introduction}

Intermittent jets injected into a cavity are rather common in widely different applications; ranging from the injection of blood into and from the heart ventricles to the fuel injection into internal combustion engines. Understanding their behaviour is becoming more and more crucial. As an example, heavy duty vehicles (HDV) and cars are responsible for around 5\% (European Commission 2007) and 12\% (European Commission 2014) respectively of total EU emissions of carbon dioxide $\left(\mathrm{CO}_{2}\right)$, the main greenhouse gas. In view of these facts, the European Commission pursues firmly the policy of reducing the environmental impact of transports and disciplining automotive engines emission requirements. This is one of the reasons why engines innovation, seeking to improve their performance and efficiency, has become a key task in mechanical engineering, technology and research.

Combustion in modern internal combustion engines is a complex phenomenon that depends on the mixing of the fuel with air and is associated with turbulent conditions. Turbulence may intensify combustion but it may also quench the flame. In the case of spray combustion, the injection process is the phase which has most potential to improve combustion, by enhancing mixing in the short time between injection and combustion. In addition, this mixing by pulsed injection is relevant also in several different areas, such as liquid/liquid and gas/liquid dispersions in chemical and chemical engineering applications or processing and treatment of radioactive waste and in general pollutant dispersion. 
Many previous studies have clarified that a more efficient entrainment of bulk ambient fluid and an increased jet mixing rate can be attained by unsteady, e.g. pulsatile instead of continuous, injection (Tanaka 1984; Bremhorst and Hollis 1990; Johari and Paduano 1997; Nathan et al. 2006). This has led to the design of the so called common rail injectors in modern diesel engines: the injectors can be steered electronically with respect to duration and frequency of injection (Doudou 2005).

In addition, both older and recent studies (Borée et al. 1997; Musculus 2009; $\mathrm{Hu}$ et al. 2012; Grosshans et al. 2015) have shown that, during and after a deceleration phase, pulsed jets experience better mixing. Musculus (2009) has investigated analitically the genesis of a wave of increased entrainment, related to the deceleration phase, which travels downstream at twice the jet penetration rate. The simplified model was later on extended to include full numerical simulations. For example Grosshans et al. (2015) used LES in their numerical study of intermittent jet mixing. The entrainment at the head of the jet is found to be lower (in the accelerating phase) as compared to the entrainment at the tail of the injected jet during the deceleration phase. Additionally, as the initial speed of the tail of the jet is larger than the speed of its head, the shape of the jet and thereby the mixing is enhanced as the jet bulb travels downstream.

The concept of entrainment wave, as introduced by Musculus (2009), derives from a simplified version of the integral momentum transfer equation in jets

$\frac{\partial \dot{M}}{\partial t}=-2 \sqrt{\frac{\beta \dot{M}}{\rho A}} \frac{\partial \dot{M}}{\partial x}$.

It is a nonlinear first-order wave equation where $\beta$ is a positive coefficient and $\mathrm{M}$ is the integral momentum. So, a deceleration transient $\left(\frac{\partial \dot{M}}{\partial t}<0\right)$ generates a positive momentum flux spatial gradient $\frac{\partial \dot{M}}{\partial x}$ and therefore an increased entrainment rate. More details can be found in Musculus (2009).

This paper aims, through experiments, to shed light on the influence exerted on the flow field by the jet temporal profile. In particular we aim to study the effects that the deceleration phase has on the generated flow field and mixing. In a combustion engine, prior and during the combustion, there are substantial pressure and temperature gradients. Hence baroclinic and dilation effects may be equally important as vortex stretching in vorticity and turbulence dynamics. In order to isolate the latter effects, in our experiments we exclude compressibility effects, whereby only the effects of vortex stretching in the enhancement of vorticity and in the transfer of turbulent energy to smaller scales (assuming that viscous effects are small) is considered. The experimental set-up uses injection of liquid into liquid (water into water), and focuses only on the effect of the temporal shape of some injection profiles.

Intermittent injection implies the additional formation of vortex rings. Their formation dynamics can be characterized through a dimensionless timescale based on the formation process energy, i.e. $\hat{T} \approx 4$ (Gharib et al. 1998) $\left(\hat{T}=\frac{L}{D}\right.$ in a piston-cylinder apparatus) with piston motion along a length of $L$ and diameter $D$. When vortex generation durations are shorter or equal to the limiting value $(\hat{T} \leq 4)$, all the vorticity is collected by the initial, leading vortex ring. Whereas for larger injection times, all the vorticity induced starting from $\hat{T} \approx 4$ is generated at the trailing jet vortex, although unable to nourish the main vortex with additional vorticity. Krueger (2001) was able to demonstrate that such limiting time corresponded to the maximum normalized time-averaged thrust; Dabiri and Gharib (2004) found that a vortex ring can contain up to $65 \%$ of entrained fluid fraction; Olcay and Krueger (2008) revealed through a PLIF study that the time variation of injection velocity along with the formation number timescale $\hat{T}$ affects the initial and terminal entrainment rates; while Querzoli et al. (2010) related the limiting time in gradually varying velocity flows, representative of real flows in nature, to the time when the positive acceleration ends, that is when $u^{*}=\frac{L(t)}{t}$ is maximum, $u^{*}$ being the velocity of the ejected fluid and $L$ the piston stroke length.

The paper is organized as follows: Sect. 2 describes the experimental setup and the measurement techniques. Section 3 is devoted to the experimental results in terms of flow statistics, entrainment ratio and triple decomposition (phase dependent) of the velocity field. Finally, in Sect. 4 our conclusions are presented.

\section{Experimental setup}

The experimental setup includes the pumping system and the measurement system, as depicted schematically in Fig. 1. The pumping system is basically a hydraulic circuit originating from an auxiliary tank and driven by a piston-like system. The motion of the piston is driven by a programmable servo motor and can be defined arbitrarily by the user. A ballscrew mechanism transforms this rotational motion into linear and transmits it to a piston/cylinder. A fully-pulsed jet condition (only blowing and no-flow phases, no suction phase) is obtained thanks to a membrane chamber located downstream to the cylinder and equipped with check valves to dissociate the flowing water from the pumping mechanism. A flow-rectifying pipe connects this plastic chamber to the nozzle, downstream to which the pulsed jet is injected into a water tank $(18 \mathrm{~cm} \times 18 \mathrm{~cm} \times 50 \mathrm{~cm})$. Originally, this rig was constructed as a phantom setup in order to measure the diastolic function of the human heart, a more detailed description thereof can be found in Töger et al. (2015). 


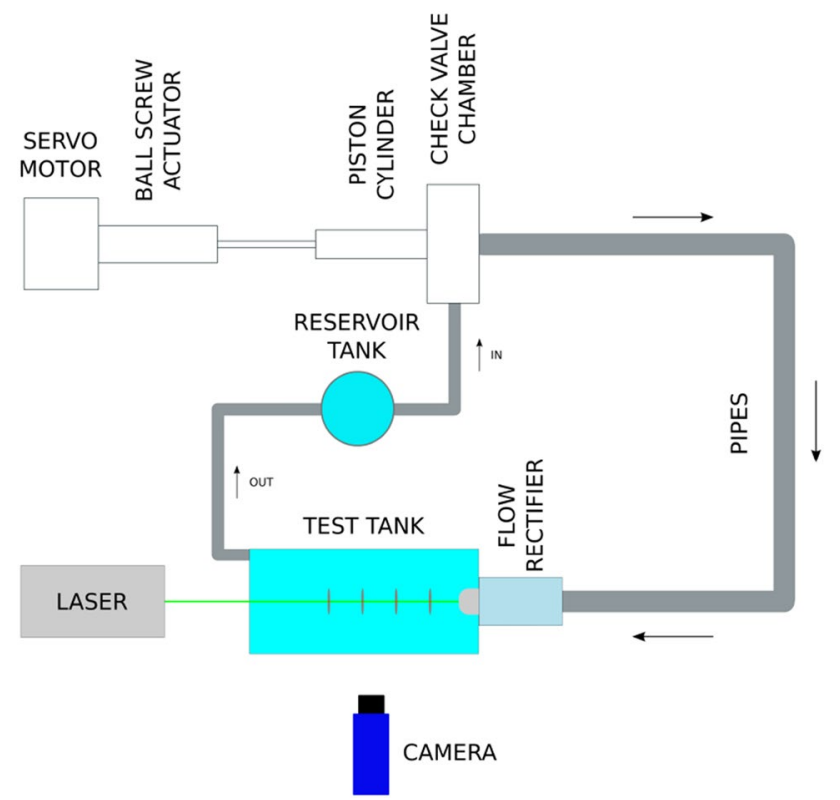

Fig. 1 Schematic of the experimental setup

Planar PIV measurements are performed in the vertical plane at the centreline of the water tank/nozzle.

PIV is a proven technique (c.f. Adrian 2005) which allows to obtain spatial information on the velocity field in a plane illuminated by a laser. Consequently the acquisition system is composed of a Nd:Yag pulsed laser ( $25 \mathrm{~mJ} /$ pulse) and of a Flowmaster 3s Camera (12 bit, 1280 pixel $\times 1024$ pixel resolution). The camera had a $6.7 \mu \mathrm{m}$ sensor pixel size and was equipped with a $50 \mathrm{~mm}$ focal length objective set at an aperture equal to $f_{\#}=2$. The water was seeded by glass hollow beads having a density of $1.05-1.15 \mathrm{~g} / \mathrm{cm}^{3}$ and a diameter around $10 \mu \mathrm{m}$. The average particle image size was around 2.52 pixels. The particle image densities, evaluated as number of particles per pixel $\left(N_{\mathrm{ppp}}\right)$, was at all times about 0.04 . These values are close to the optimal value prescribed by Willert and Gharib (1991) $\left(N_{\mathrm{ppp}}=0.035\right)$ and in agreement with the recommendations of Raffel et al. (2007) and Cierpka et al. (2013) $\left(0.03<N_{\mathrm{ppp}}<0.05\right)$. The magnification factor M was about 11 pixel $/ \mathrm{mm}$ (roughly $0.09 \mathrm{~mm} /$ pixel). The light beam generated by the laser was converted by a cylindrical and spherical lenses optics set into a light sheet having a thickness of about $1 \mathrm{~mm}$. The image mapping parameters, providing the relation between the positions $\left(x_{\mathrm{obj}}, y_{\mathrm{obj}}, z_{\mathrm{obj}}\right)$ in the object domain and the corresponding positions in the image plan $\left(x_{\mathrm{im}}, y_{\mathrm{im}}\right)$ (see Soloff et al. 1997), have been determined inserting a dual plane calibration target in the object domain (laser sheet plane), illuminated with an incoherent white light source instead of the laser, to avoid speckle pattern on the surface that compromise the contrast of the calibration image (Adrian and Westerweel 2011). The locations of the target's markers are reconstructed by a cross-correlation operation between calibration and template images in addition to a peak-finding algorithm (Willert 2006).

Acquired images have been processed by a digital crosscorrelation analysis (Willert and Gharib 1991). A multi-grid/ multi-pass algorithm (Soria 1996), with an iterative image deformation (Scarano 2001; Huang et al. 1993; Jambunathan et al. 1995; Nogueira et al. 1999) was used to compute the instantaneous velocity fields. The algorithm was based on two steps on a larger window size $(64 \times 64$ pixels $)$ and four steps on a smaller $(32 \times 32$ pixels $)$ with an overlap factor of $50 \%$. Moreover, to eliminate spurious vectors, a vector validation algorithm, based on a regional median filter ( Westerweel and Scarano 2005), with kernel region of $3 \times 3$ vectors, and group removing, is applied.

It has been shown that the size of the framed area can affect PIV results. For a fully developed turbulent flow, it was discovered that the size of the acquired region should not exceed a limiting value around 40 Taylor microscales (Lacagnina and Romano 2015). This condition depends on the size of the array used to record the PIV images, so the same limit value is not applicable in this case. Moreover, in the current measurements, the flow is not turbulent intermittently and even then it is not fully developed. Anyhow, one may estimate the "Taylor microscale" $\lambda$ in this situation by the auto-correlation function. This estimate gives a value of approximately $\lambda=3.2 \times 10^{-3} \mathrm{~m}\left(R e_{\lambda} \approx 650\right)$ and therefore the framed area $(11 \mathrm{~cm} \times 9 \mathrm{~cm})$ is slightly lower than the limit $40 \lambda$.

\section{Results}

In this study three different time-dependent injection strategies have been chosen (as shown in Fig. 2), and they differ in terms of how the velocity changes along the pulse cycle. The first one is a square (on/off) profile characterized by extremely strong acceleration and deceleration, separated by a constant injection velocity phase. This strategy is depicted by the ratio between two time-sequences: namely the injection time $T_{1}$ and the rest (no-injection) time $T_{2}$. A value of $\frac{T_{1}}{T_{2}}=0.5$ has been chosen as reference, while the values 0.2 and 0.85 have been studied for comparison. The second injection strategy is based on an isosceles triangular velocity profile in time, with an acceleration phase equal in modulus to the deceleration phase. Finally a scalene triangular velocity profile, having a deceleration larger in modulus (stronger) as compared to the acceleration (LAHD, low acceleration high deceleration). The three injection schemes have the maximum velocity at the cylinder in common and it is equal to $0.3 \mathrm{~m} / \mathrm{s}$. Obviously, the largest acceleration/deceleration occurs in the square (on/off) case and the mildest 


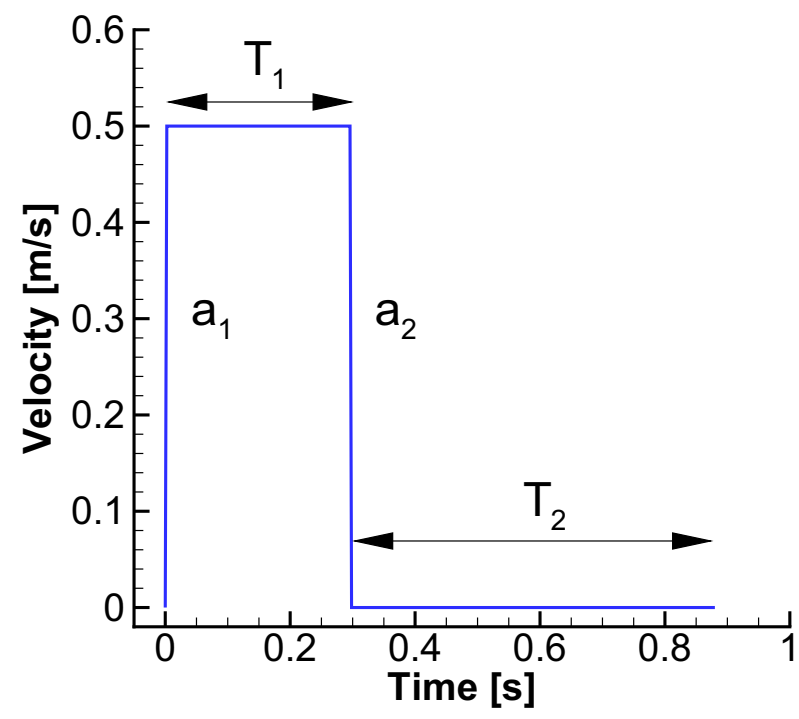

(a) Square

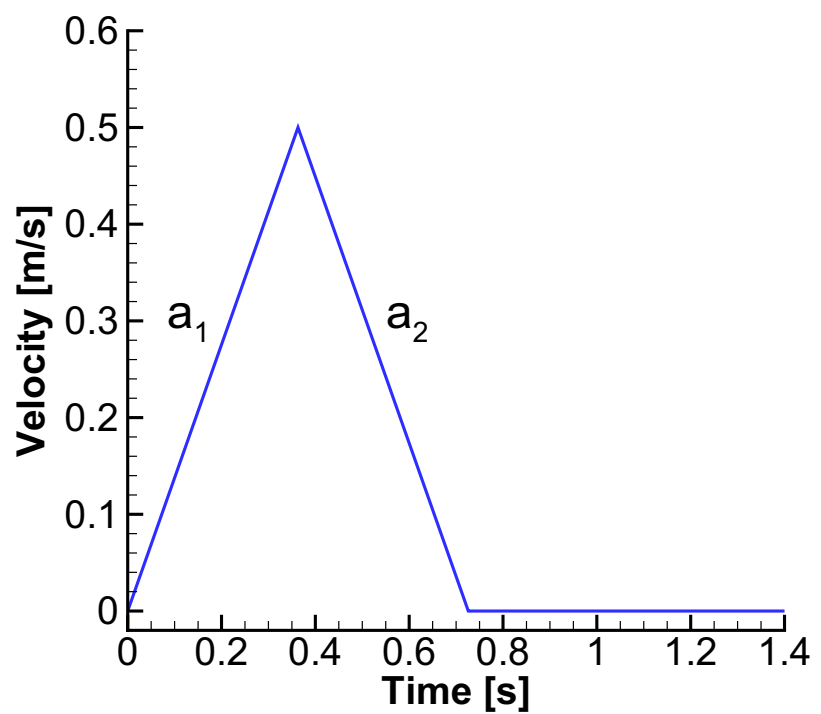

(b) Isosceles

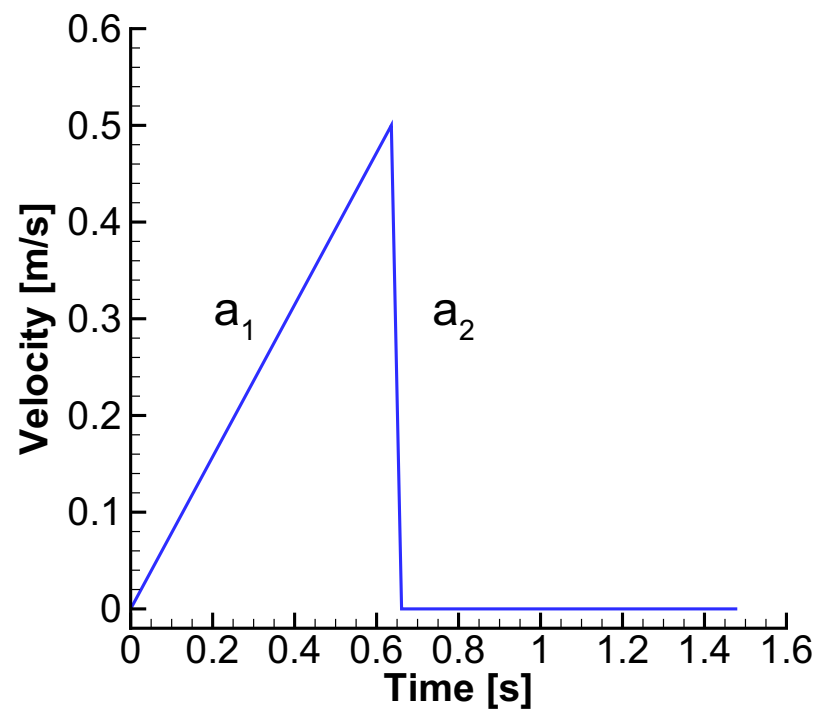

(c) LAHD

Fig. 2 Velocity profiles of the different injection strategies: a square injection, $\mathbf{b}$ isosceles triangle injection, $\mathbf{c}$ low acceleration high deceleration (LAHD) injection

deceleration is in the isosceles triangular case, see Table 1. The injection profiles defined here vary in the injected momentum/mass. However, the different injection profiles have in common the mass flow rate, being close to $0.08 \mathrm{~g} / \mathrm{s}$, and the volume flow rate, in the range $4-5 \mathrm{~L} / \mathrm{min}$, as shown in Table 1. These values are not so different from the real ones for high duty diesel engine fuel injectors $(2-2.5 \mathrm{~L} / \mathrm{min})$ and anyhow constrained from our setup. Moreover, as we are focusing on the mixing, we can assume that the required amount of injected fluid or its momentum, can be accounted
Table 1 Features of the velocity profiles

\begin{tabular}{lllll}
\hline Profile & $\begin{array}{l}\text { Accelera- } \\
\text { tion }\left(\mathrm{m} / \mathrm{s}^{2}\right)\end{array}$ & $\begin{array}{l}\text { Decelera- } \\
\text { tion }\left(\mathrm{m} / \mathrm{s}^{2}\right)\end{array}$ & $\begin{array}{l}\text { Mass flow } \\
\text { rate }(\mathrm{g} / \mathrm{s})\end{array}$ & $\begin{array}{l}\text { Volume flow } \\
\text { rate }(\mathrm{L} / \mathrm{min})\end{array}$ \\
\hline $\begin{array}{l}\text { Isosceles } \\
\begin{array}{c}\text { High decelera- } \\
\text { tion (LAHD) }\end{array}\end{array}$ & 0.79 & 1.38 & 0.089 & 5.35 \\
$\begin{array}{l}\text { Square } \\
\text { quan }\end{array}$ & 200 & 200 & 0.081 & 4.87 \\
\hline
\end{tabular}


for by, for example, multiple injectors. Here, we aim at comparing different injections strategies with given injection rates during a given injection time.

Moreover, in order to study the effect of the Reynolds number on jet features, three different nozzle diameters have been installed: $D_{0}=25 \mathrm{~mm}, D_{1}=\frac{D_{0}}{2}=12.5 \mathrm{~mm}$ and $D_{2}=\frac{D_{0}}{3}=8.3 \mathrm{~mm}$. Keeping the volume flow rates constant, the variation in the cross section is balanced by an increased inlet velocity corresponding to Reynolds numbers in the range $R e=11,000-34,000$, see Table 2 .

The measurement section was located two $D_{0}$ diameters downstream to the nozzle edge on its symmetry axis $(x$, see Fig. 3) and it extends along roughly four diameters $D_{0}$. The origin of the coordinate system is in center of the nozzle section, in correspondence to its lip. Therefore the $u$ velocity component is along the streamwise direction ( $X$ axis) of the jet and the $v$ component along the vertical direction ( $Y, r$ axis). Each injection cycle was subdivided into twenty periods and fifty PIV images (double frame) were acquired in correspondence to each phase.

\subsection{Effect of design strategy}

The first effect to be studied was the one due to the way velocity changed during injection. Three representative cases, corresponding to the three different injection profiles have been chosen. Figure 4 shows the evolution in time of the mean velocity at the most upstream location of the framed area. As can be seen, the high deceleration strategy $L A H D$ experiences an acceleration in the first sub-periods while the remaining two are already experiencing the deceleration common in the travelling of an established jet. This is due to the fact that, at the moment when the $L A H D$ vortex enters into the framed area, its injection is still ongoing due to the low acceleration.

As can be seen in Fig. 5, which identifies the moment into the injection scheme when the vortex enters in the framed area, at this time the $L A H D$ scheme is still in the acceleration phase and has not reached its maximum velocity. Identifying

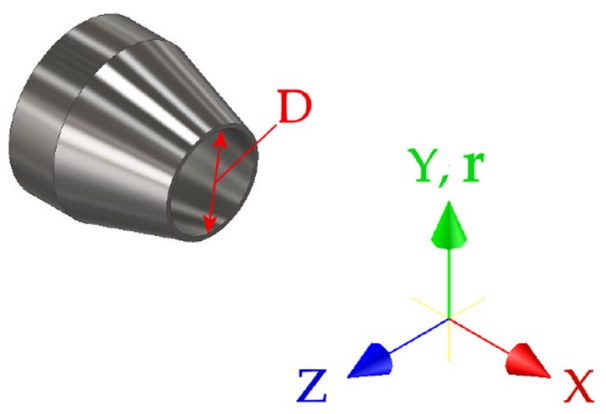

Fig. 3 Nozzle dimensions and coordinate system. The measurement plane lies in the plane $(X, Y(r))$

the vortex position by the location of vorticity maxima, the travel of vortices into the framed area can be tracked. It is evident how the $L A H D$ vortex travels more slowly compared to the latter two. The slope of the positions evolution enlightens the lower velocity of $L A H D$ in the first periods, while the three velocities become comparable in the following periods.

A large portion of mixing in pulsed jets is related to the strong entrainment in correspondence to the tail of the jet. Investigating the radial velocity component can be a good tool to quantify this effect and to compare the mixing performance of the different injection rationales. Figure 6 depicts the time-development of the radial velocity as isocontours in the $y$-cross section at $x=4 D$. A positive radial velocity is observed in the first stages of each injection scheme, evidencing the expansion of the jet. This condition is followed by a radial flow reversal, just after the jet has passed the sampling location $(x=4 D)$, resulting in a strong radial inflow towards the jet axis. It is evident how the $L A H D$ scheme generates a wider area (in the timeradial position subspace) of negative (entraining) radial velocity as compared to the other ones, while a higher local amplitude of negative velocity is achieved by the square profile. Performing an integration along the area (in the time-radial position subspace) of negative velocity allows to quantitatively compare the three schemes. Values

Table 2 Measured cases

\begin{tabular}{llllll}
\hline Profile & $\begin{array}{l}\text { Piston } \\
\text { velocity } \\
(\mathrm{m} / \mathrm{s})\end{array}$ & $\begin{array}{l}\text { Nozzle diam- } \\
\text { eter }(\mathrm{mm})\end{array}$ & $\begin{array}{l}\text { Outlet } \\
\text { velocity } \\
(\mathrm{m} / \mathrm{s})\end{array}$ & $\begin{array}{l}\text { Jet cycle } \\
\text { period }(\mathrm{s})\end{array}$ & Reynolds number \\
\hline Isosceles & 0.305 & 25 & 0.5 & 1.4 & 11,000 \\
High deceleration (LAHD) & 0.305 & 25 & 0.5 & 1.48 & 11,000 \\
Square $\frac{T_{1}}{T_{2}}=0.5$ & 0.305 & 25 & 0.5 & 0.88 & 11,000 \\
Square $\frac{T_{1}}{T_{2}}=0.2$ & 0.305 & 25 & 0.5 & 1.94 & 11,000 \\
Square $\frac{T_{1}}{T_{2}}=0.85$ & 0.305 & 25 & 0.5 & 0.7 & 11,000 \\
Isosceles $\frac{D_{0}}{2}$ & 0.305 & 12.5 & 2 & 1.4 & 22,500 \\
Isosceles $\frac{D_{0}}{3}$ & 0.305 & 8.3 & 4.5 & 1.4 & 34,000 \\
\hline
\end{tabular}




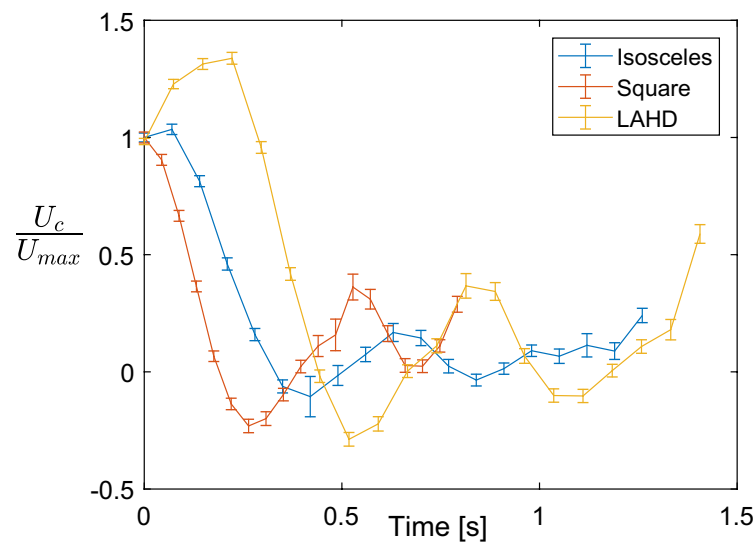

(a)

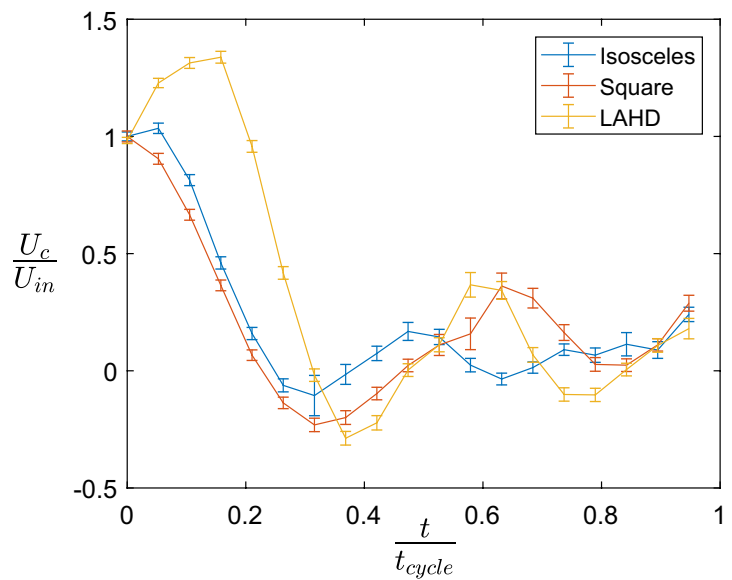

(b)

Fig. 4 Temporal evolution of the measured mean axial velocity at $x=2 D$ for the isosceles injection strategy (blue line), square injection strategy (red line), $L A H D$ injection strategy (yellow line). Errorbars show the RMS of results. a Dimensional time, b time normalized by cycle time

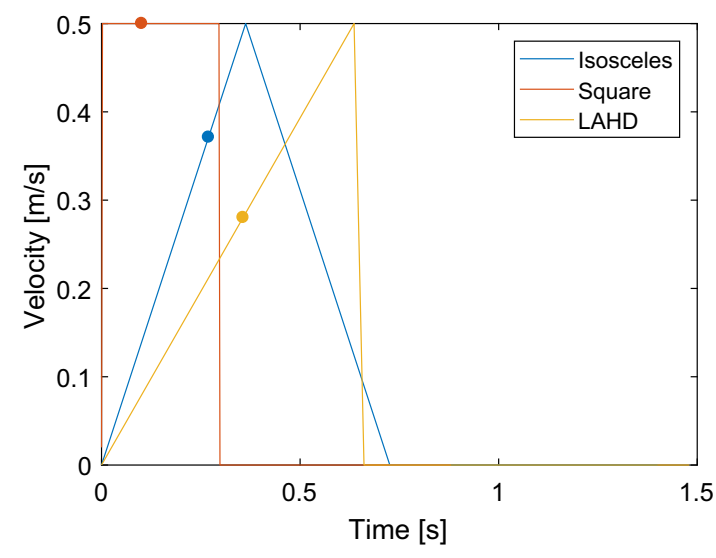

(a)

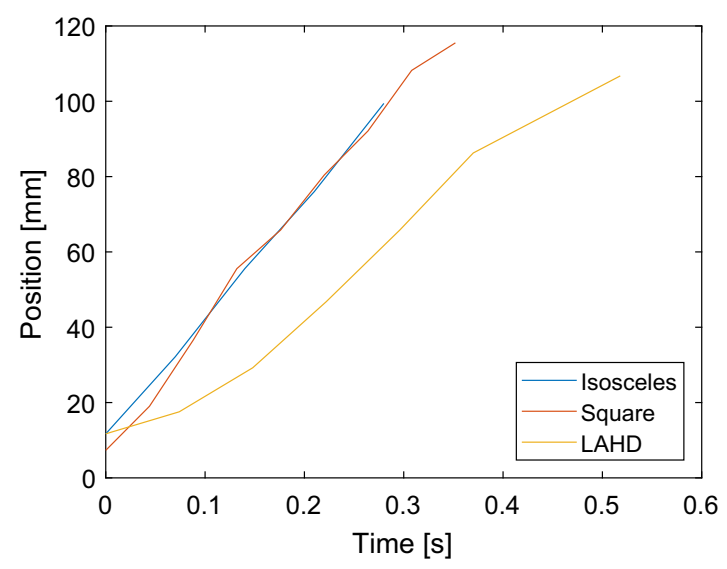

(b)

Fig. 5 a Time when vortices enter the framed area. $\mathbf{b}$ Time evolution of vortex position starting from $x=2 D$ downstream to the nozzle. Legend colours are: blue line for the isosceles injection strategy, red line for the square injection strategy and rellow line for the LAHD injection strategy

$0.14,0.23,0.33$ correspond to the isosceles, LAHD and square profiles, respectively. Therefore it can be stated that a more intense deceleration is able to increase the entrainment among triangular profiles while the square profile, embodying the strongest deceleration phase, achieves the highest local entrainment.

A complementary way of assessing the mixing ability of a phenomenon is estimating the variation of the entrainment ratio $(E)$ during its evolution. It is defined by Wygnanski and Fiedler (1969), Crow and Champagne (1971) and Liepmann and Gharib (1992) as the spatial derivative of the mass flow rate $Q$ in the streamwise direction $\left(\frac{\mathrm{d} Q}{\mathrm{~d} x}\right)$, or conversely as its deficit with respect to its amount at the inlet location $Q_{0}$ compared to the $Q_{0}$ itself $\left(E=\frac{Q-Q_{0}}{Q_{0}}\right)$. The mass flow rates $Q$ were numerically estimated integrating the samples mean streamwise velocity at each subperiod of the pulse cycle making use of the definition (Quinn et al. 2012):

$Q=\int_{A} \rho U \mathrm{~d} A$,

where $\mathrm{d} A$ is an elemental area in the $x-y$ plane, evaluated assuming the axisymmetry of the phenomenon. In correspondence to all the streamwise locations, the integration was carried out until the radial location where the samples mean streamwise velocity was equal to the $10 \%$ of the corresponding centerline value, c.f. (Quinn et al. 2012). In this way, by integrating only the core of the jet, and by assuming 


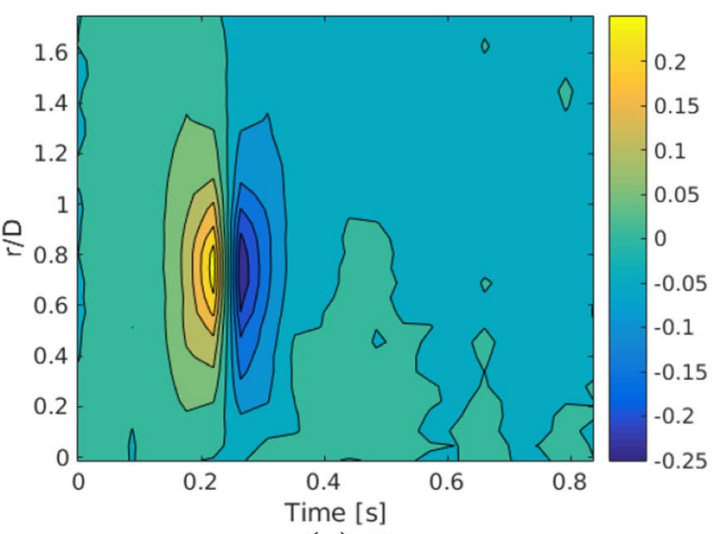

(a) Square

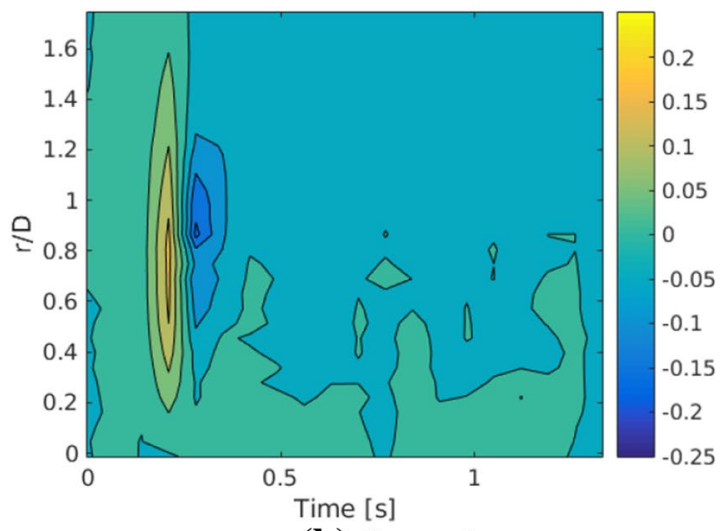

(b) Isosceles

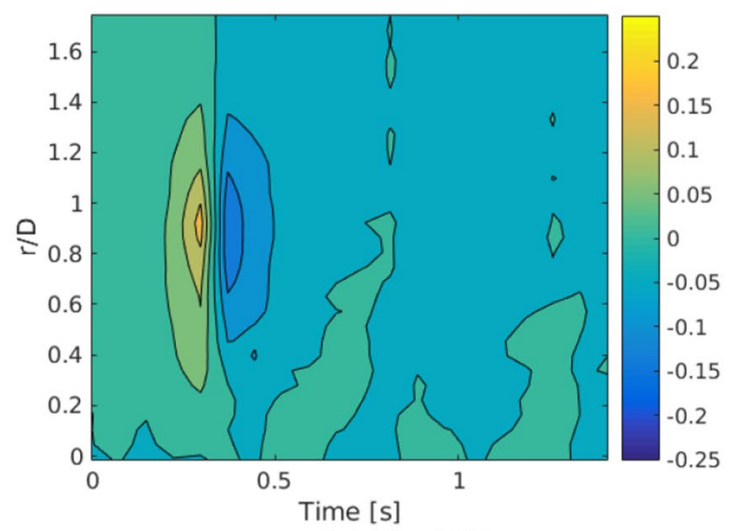

(c) LAHD

Fig. 6 Mean radial velocity temporal evolution at $x=4 D$ for a square injection strategy, $\mathbf{b}$ isosceles injection strategy, $\mathbf{c}$ LAHD injection strategy. Note that only half of the vertical plane $r$ is depicted, assuming rotational symmetry. The units are $\mathrm{m} / \mathrm{s}$

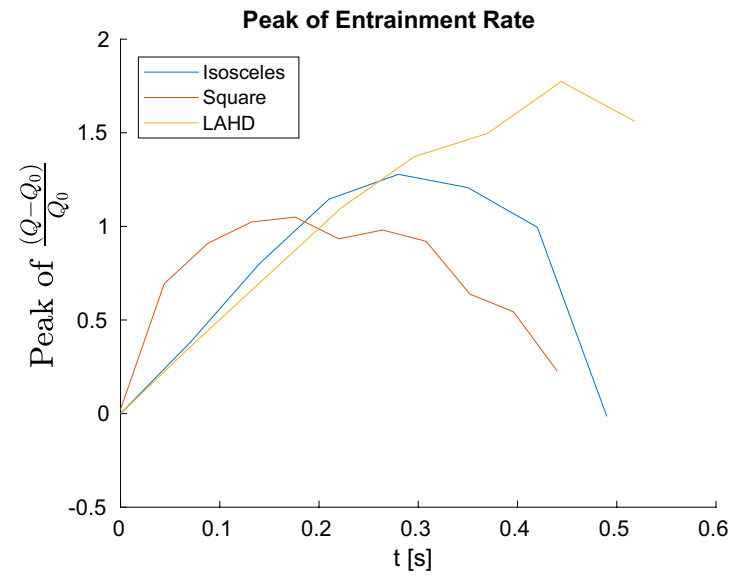

(a)

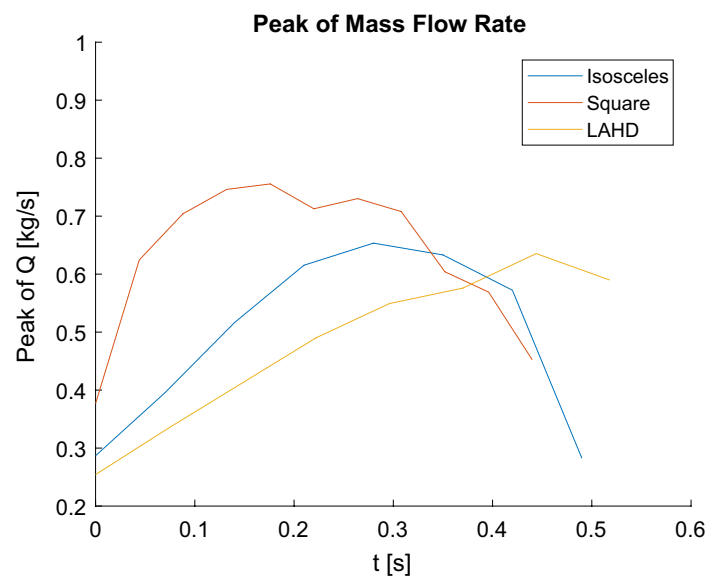

(b)

Fig. 7 Comparison of the different injection strategies [isosceles injection strategy (blue line), square injection strategy (red line), LAHD injection strategy (yellow line)] in terms of: a peaks of entrainment ratios. b Peaks of mass flow rate 
conservation of momentum, $E$ can be seen as a metric of mixing, since it assesses the mass entrained by the jet from the ambient fluid.

Next, consider the maximum entrainment at each subperiod of the pulse cycle, which is depicted in Fig. 7. Here $Q_{0}$ is the mass flow rate when the vortex enters the framed area. It is evident how the symmetrical triangular profile (isosceles) rapidly reaches a high value which is however followed by a rapid decay. The $L A H D$ in comparison is able to maintain a good high level of entrainment for a longer period of time. This can be ascribed to the entrainment wave associated with the deceleration phase, which is able to sustain the amount of mass entrainment.

In a similar way one may compare the different square injection strategies, namely $\frac{T_{1}}{T_{2}}=0.2, \frac{T_{1}}{T_{2}}=0.5$ and $\frac{T_{1}}{T_{2}}=0.85$, see Fig. 8. From this plot the shortest "injection to rest" ratio strategy $\left(\frac{T_{1}}{T_{2}}=0.2\right)$ seems to generate an entrainment fairly higher than the other cases. However, plotting the mass flow rate by itself (Fig. 8 right) it is evident how this result is influenced by the different inflow conditions. In other words, for low $\frac{T_{1}}{T_{2}}$ the water has more time to settle down and therefore the mass flow rate at the vortex arrival time, $Q_{0}$, is substantially lower than the one for the other cases, nevertheless the mass flow rate for $\frac{T_{1}}{T_{2}}=0.2$ becomes quickly higher than for the remainders.

Concurrently, tracking the vortex position, for different square injection strategies, by the position of vorticity maxima, as in Fig. 5 left, it can also be observed how the lower the ratio $\frac{T_{1}}{T_{2}}$ the faster the vortex travels, see Fig. 9. This means that, for the smaller ratio $\frac{T_{1}}{T_{2}}$, not only the the vortex is able to produce an higher entrainment, but it also

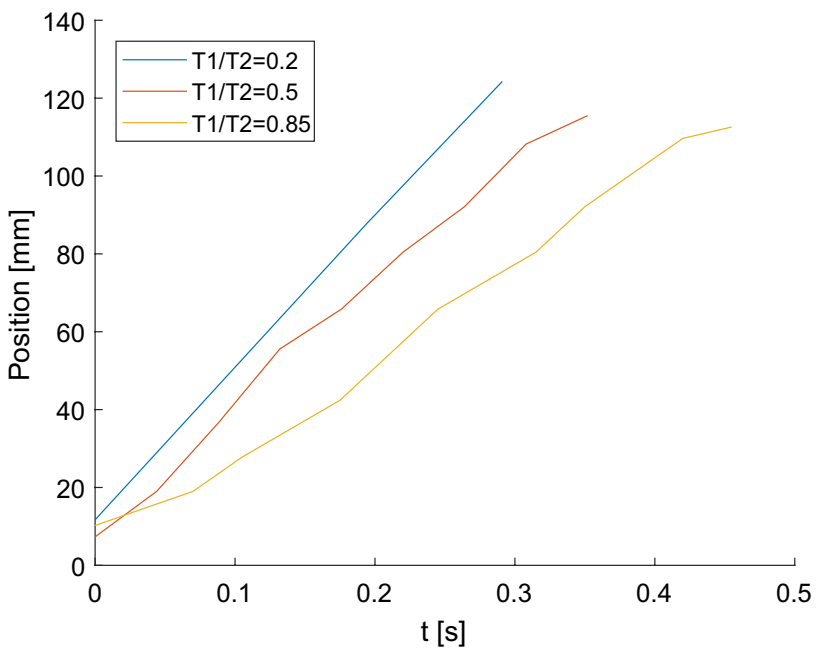

Fig. 9 Time evolution of vortex position for square injections [ $T_{1} / T_{2}=0.2$ (blue line), $T_{1} / T_{2}=0.5$ (red line), $T_{1} / T_{2}=0.85$ (yellow line)], starting from $x=2 D$ downstream to the nozzle

more easily can make its way in a smoother surrounding fluid (when the fluid has had more time to settle).

Next, we consider the entrainment rate as function of velocity (Reynolds number) and Fig. 10 shows the temporal evolution of the peaks of entrainment ratio. In these cases the original nozzle $D=D_{0}=25 \mathrm{~mm}$ was replaced by two smaller ones with diameters of $D_{1}=\frac{D_{0}}{2}=12.5 \mathrm{~mm}$ and $D_{2}=\frac{D_{0}}{3}=8.3 \mathrm{~mm}$, respectively. Thereby the mean velocity increased from 0.5 to 2 and $4.5 \mathrm{~m} / \mathrm{s}$, respectively, as the volume flow rate is kept constant. Hence, the Reynolds number in these cases is within the range $R e=11,000-34,000$. Further details about the different measured cases are given in Table 2. From Fig. 10 it can be inferred that the higher the

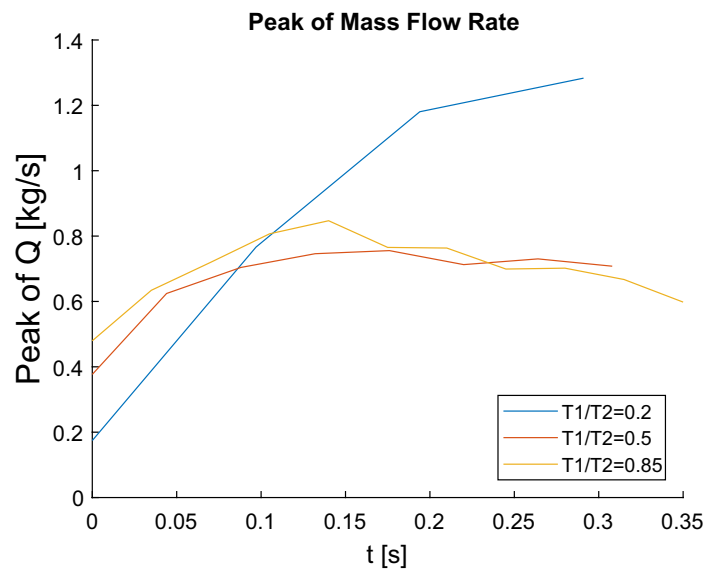

(b)

Fig. 8 Comparison between the different square injection strategies $\left[T_{1} / T_{2}=0.2\right.$ (blue line), $T_{1} / T_{2}=0.5$ (red line), $T_{1} / T_{2}=0.85$ (yellow line) , in terms of: a peak of entrainment ratios. b Mass flow rate 


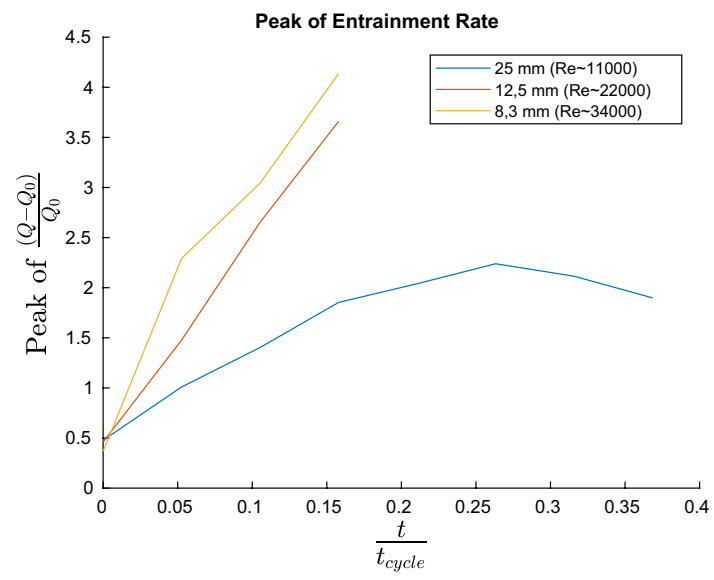

(a)

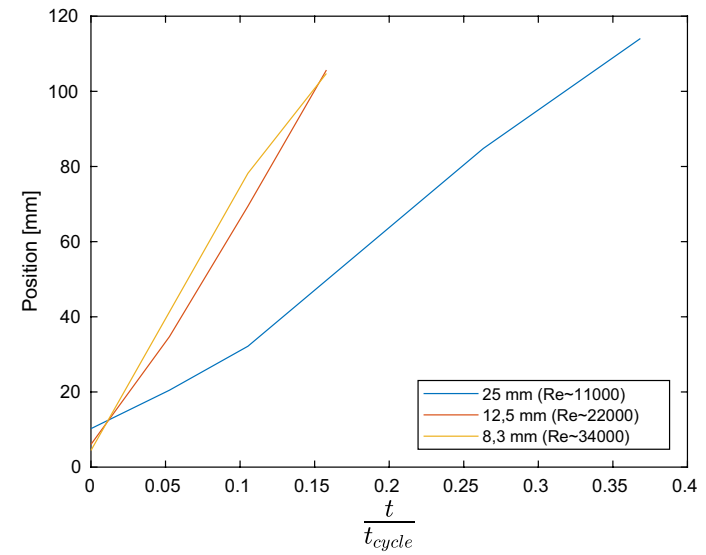

(b)

Fig. 10 Time evolution of $\mathbf{a}$ intensity and $\mathbf{b}$ location (from $x=2 D$ downstream to the nozzle) of the peak of entrainment ratios for different $R e$ numbers [ $R e \approx 11,000$ (blue line), $R e \approx 22,000$ (red line), $R e \approx 34,000$ (yellow line)]

Reynolds, the greater is the entrainment achievable, at least in this range of experimental conditions.

\subsection{Triple decomposition}

A common tool to study a turbulent flow is the so called "Reynolds decomposition", introduced by Reynolds (1895) and based on the temporal mean of the velocity. The fluctuation are defined by subtracting the mean from the instantaneous values. The root mean square of the fluctuations is then a common metric for turbulence intensity. However, it is justified as an approach only as long as turbulence is the only source of fluctuations, otherwise it can lead to an overestimation of the random component of the flow, as it is the case with an intermittent jet. Hussain and Reynolds (1970) have introduced a suitable tool in this instance, namely the Triple Decomposition, beneficial in isolating organized systematic variations in velocity which should not be taken in account when investigating the chaotic or turbulent flow. According to the triple decomposition, the flow field can be decomposed as:

$u_{i}(x, t)=U_{i}(x)+\tilde{u}_{i}(x, t)+u_{i}^{\prime}(x, t)$,

where $U_{i}$ is the time average, $\tilde{u}_{i}$ is the phase-organized contribution to the velocity and $u_{i}^{\prime}$ is the turbulence velocity fluctuation contribution.

The time average is defined as

$U_{i}(x)=\lim _{T \rightarrow \infty} \frac{1}{T} \int_{0}^{T} u_{i}(x, t) \mathrm{d} t$.

And the phase average $\left(\left\langle u_{i}\right\rangle=U_{i}+\tilde{u}_{i}\right)$ is

$\left\langle u_{i}(x, t)\right\rangle=\lim _{N \rightarrow \infty} \frac{1}{N} \sum_{n=0}^{N-1} u_{i}(x, t+n \tau)$, where $\tau$ is the period of the organized contribution, which in this study is taken to be the period of the pulsed jet. The phase-averaged velocity is thus the average, at any point in space, of the velocity at a particular phase $\phi$ in the cycle of intermittent jet generation (Hussain and Reynolds 1970). Then the phase-correlated velocity contribution is given by $\tilde{u}_{i}(x, t)=\left\langle u_{i}(x, t)\right\rangle-U_{i}(x)$.

And the turbulence velocity fluctuation contribution is $u_{i}^{\prime}(x, t)=u_{i}(x, t)-\left\langle u_{i}(x, t)\right\rangle$.

One important property of this decomposition is that, on the average, the turbulence fluctuation and the phase organized motion are uncorrelated (Hussain and Reynolds 1970).

Figure 11 shows a comparison between the time averaged (across the pulsing cycle) profiles along centreline of the phase-organized streamwise velocity (left) and of the turbulence streamwise velocity fluctuations (right). It can be clearly seen that, at each location, the magnitude of turbulence related contributions is much higher than the one related to the phase. Including also the turbulence fluctuations of a continuous contoured nozzle jet, taken from Quinn (2006) and having a higher $\operatorname{Re}(\sim 180,000)$, two observations can be made. First of all, in a comparison between pulsed and continuous jets, the intermittent jet exhibits higher turbulence intensities. Since small scale motions become excited as turbulence intensity increases (Deissler 1958) and so the turbulent mixing intensifies, being triggered most efficiently by small scale motions, which ensures the closest contact between the fluid entering a region and the fluid already there (Deissler 1998), it is evident how an intermittent jet is more efficient as compared to a continuous jet in terms of mixing, at least in the turbulent shape. In addition, it can be stated that the magnitude of phase-organized 


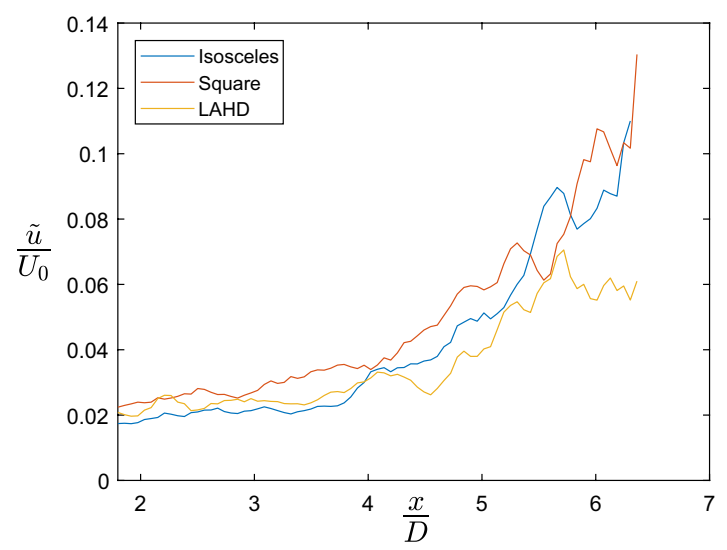

Fig. 11 Comparison between the different injection strategies [isosceles injection strategy (blue line), square injection strategy (red line), $L A H D$ injection strategy (yellow line)], in terms of: left: phase-organized fluctuations. Right: turbulence fluctuations. The spatial evolution

fluctuations is comparable to the turbulent fluctuations of a continuous jet.

By comparing the different injections strategies, it has been found that the isosceles has a lower turbulence intensity but it recoups moving downstream where the "high deceleration" strategy reduces the magnitude of both the phaseorganized and the turbulent fluctuations.

A comparison between the triple decomposition components in the whole measurement domain has then been carried out. Figures 12, 13 and 14 display the isocontours of the phase-organized and turbulent fluctuations. The axial velocity phase-correlated oscillation $\tilde{u}$, in Fig. 12a, c, e shows that the isosceles scheme has the highest phase-coherent fluctuations while the square scheme has a larger transversal extent of the shear region and the $L A H D$ has a long area of low oscillation level, up to $\left(\frac{x}{D}=4\right)$, in correspondence to the core of the vortex ring.

Plotting the random turbulent velocity fluctuations (Fig. 12b, d, f) highlights some differences between the injection strategies. High levels of fluctuations are usually confined in the nearest field in correspondence to the cores of vorticity of the vortex rings. The triangular schemes (isosceles and $L A H D$ ) show a long low fluctuation core, while the square one peaks its fluctuation level in the far field, possibly in concurrence with the breaking up of the vortex ring. Finally, the high deceleration profile displays higher coherence and less instabilities as compared to the others. As a final comment, it could be stated that the turbulent fluctuations are comparable to the phase-coherent ones although always greater in magnitude.

Figure 13 displays the phase-coherent and turbulent radial velocity fluctuations. As concerns the phase-organized ones, the $L A H D$ scheme highlights the lowest values while the

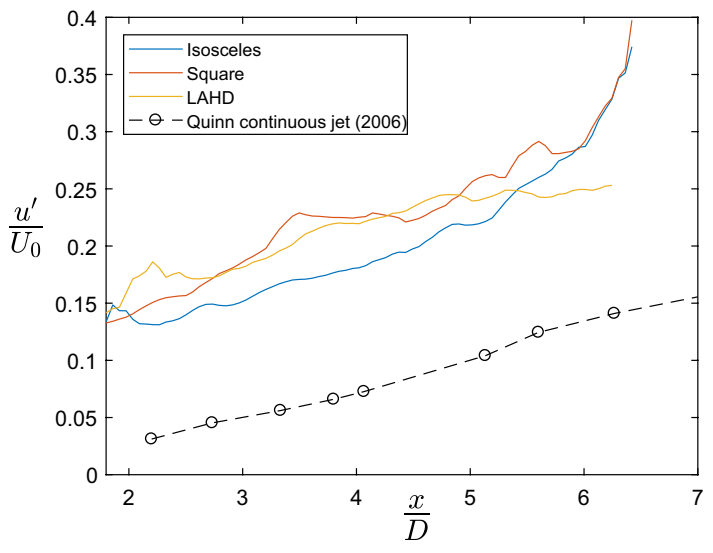

is evaluated in correspondence to the axis of the jet and the fluctuations are non-dimensionalised by the velocity when the vortex enters the framed area $U_{0}$

remaining two display peaks in the shear zone, the isosceles scheme having high values along all the jet axis, which in the square are restricted only to the near field. As regards the turbulent fluctuations, the comments are similar to the axial case: the square scheme has the highest fluctuations and restricted to the far field, while the triangular schemes have a long low fluctuation core, the $L A H D$ having a wider and less ragged shear region.

Finally, we consider the Reynolds stress, both in the phase-correlated and turbulent uncorrelated contribution. As regards the phase-coherent part, the isosceles strategy produces the highest magnitude along the entire measurement domain, clearly displaying an alternate pattern, while the $L A H D$ and square schemes have a strong peak concentrated in the near field in correspondence to the centre of the two footprints obtained by the cross-section of the vortex ring.

Finally, the turbulent Reynolds stress displays the typical outline for a turbulent jet with the two opposite sign shear layers spreading out and tending to merge towards the centre of jet. It can be stressed out that the turbulent Reynolds stress is slightly smaller than the phase correlated one.

\section{Conclusions}

This work is focused on an experimental study on the influence that the design of injection strategies have on the flow field and mixing potentiality of pulsed jets, paying a specific attention on the deceleration phase of the forcing. The attention is on the modifications of the velocity field investigated by means of the particle image velocimetry (PIV) technique. Three different injection velocity strategies have been used, namely square, isosceles triangle and high deceleration scalene triangle ( $L A H D$ ). It has been 


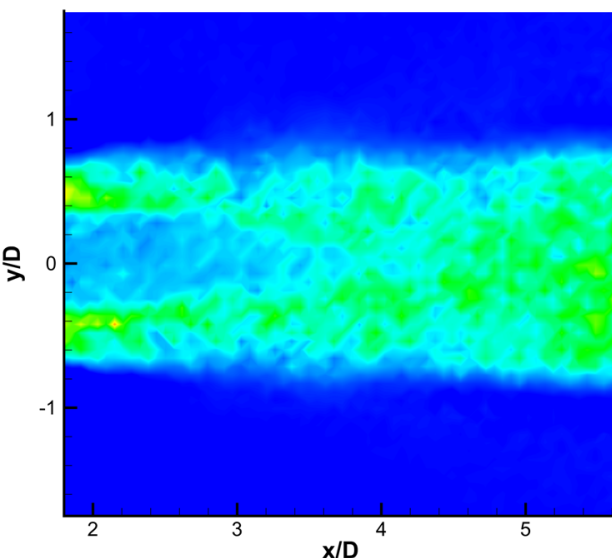

(a)

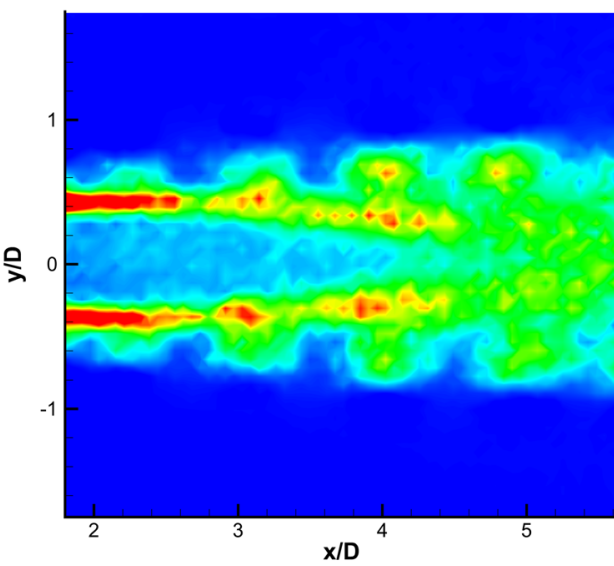

(c)

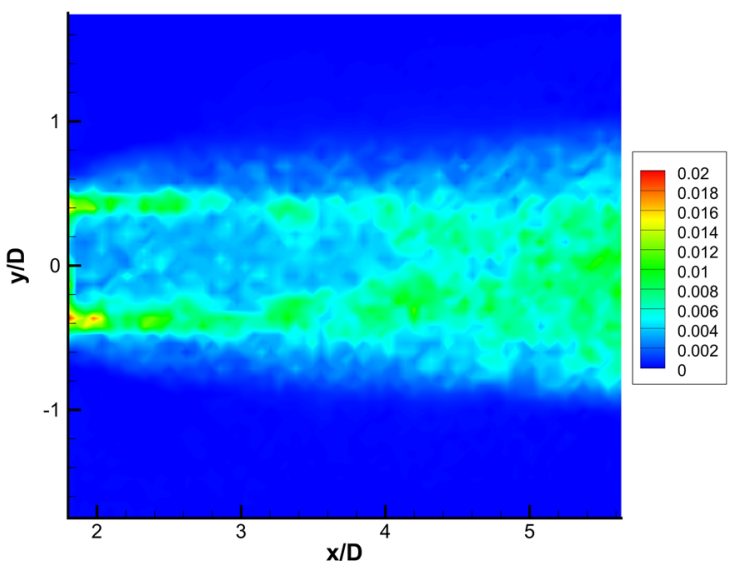

(e)

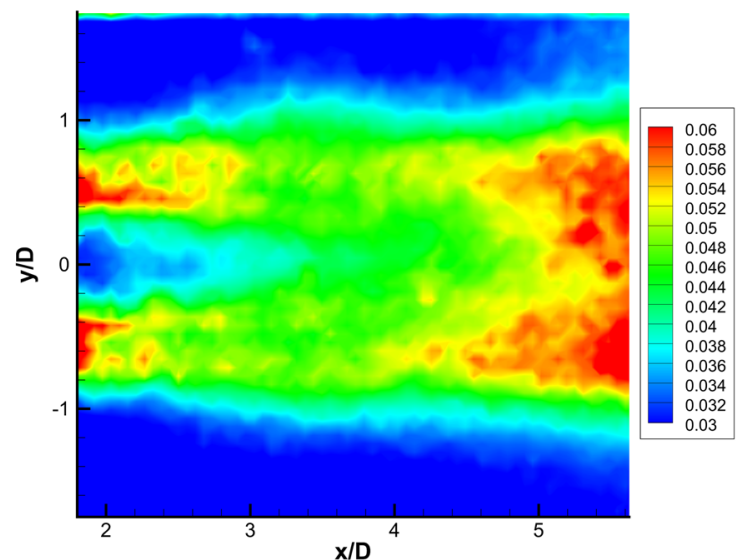

(b)

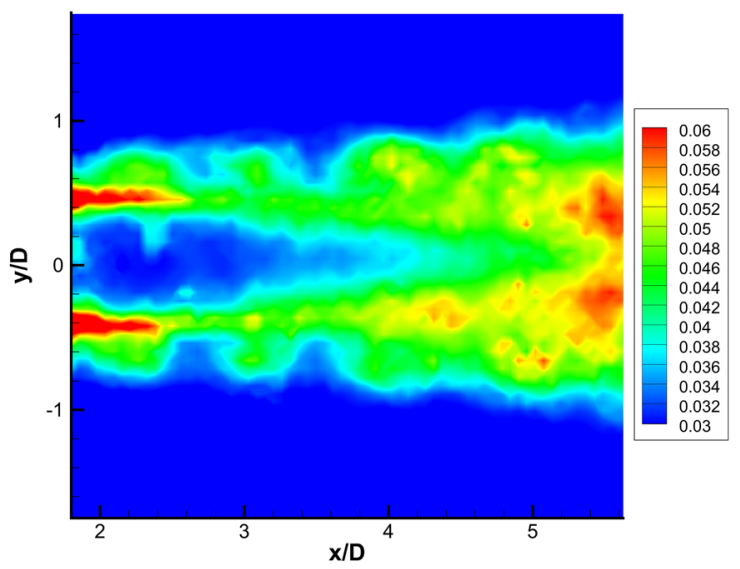

(d)

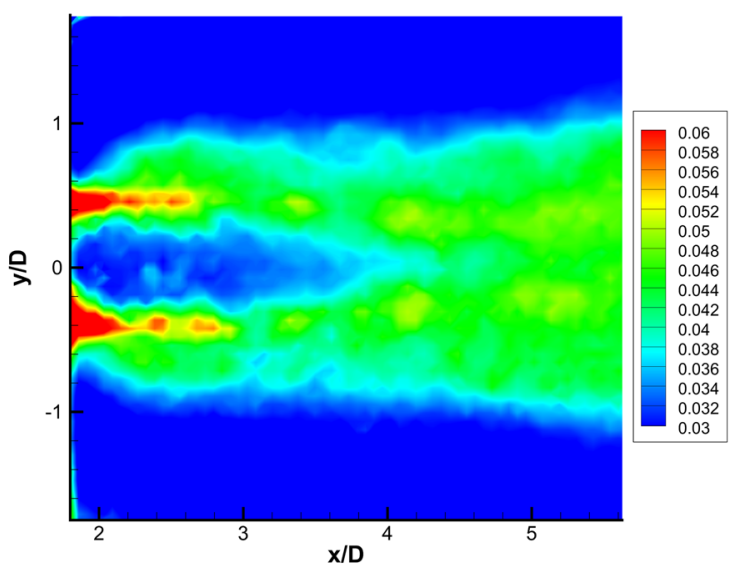

(f)

Fig. 12 Comparison between: left: phase-correlated axial fluctuation field; right: turbulent axial fluctuation field. a, b Square injection strategy; c, $\mathbf{d}$ isosceles injection strategy; e, f LAHD injection strategy. The units are $(\mathrm{m} / \mathrm{s})$ 


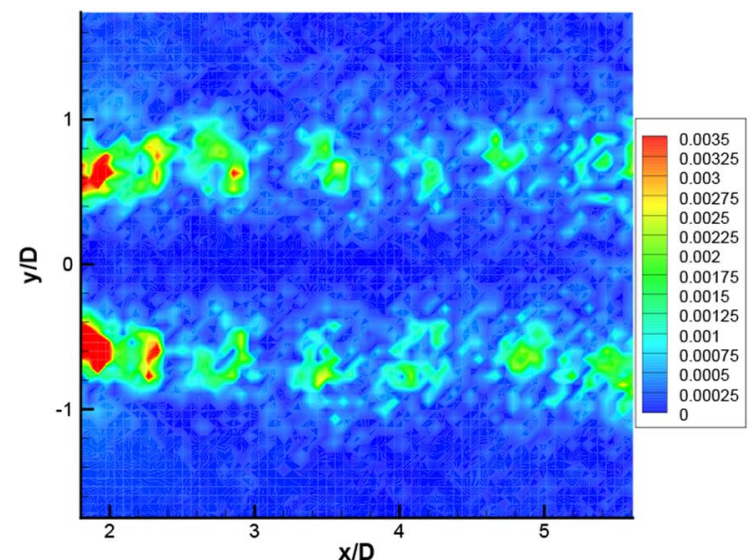

(a)

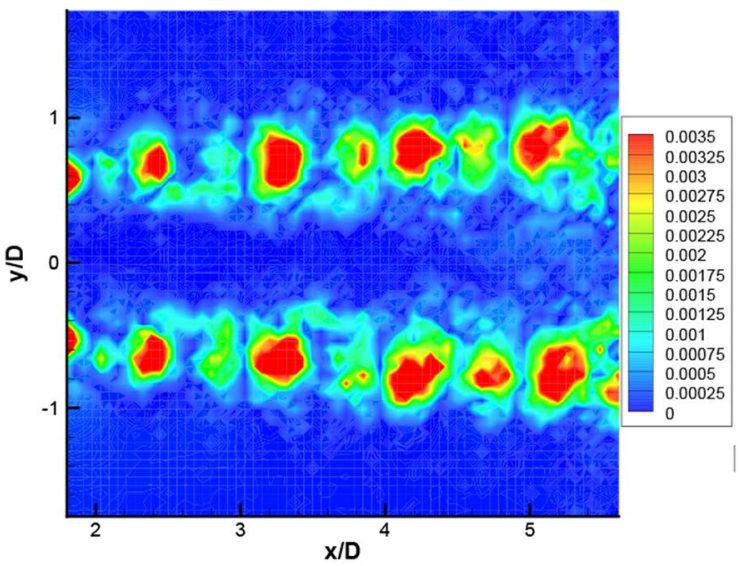

(c)

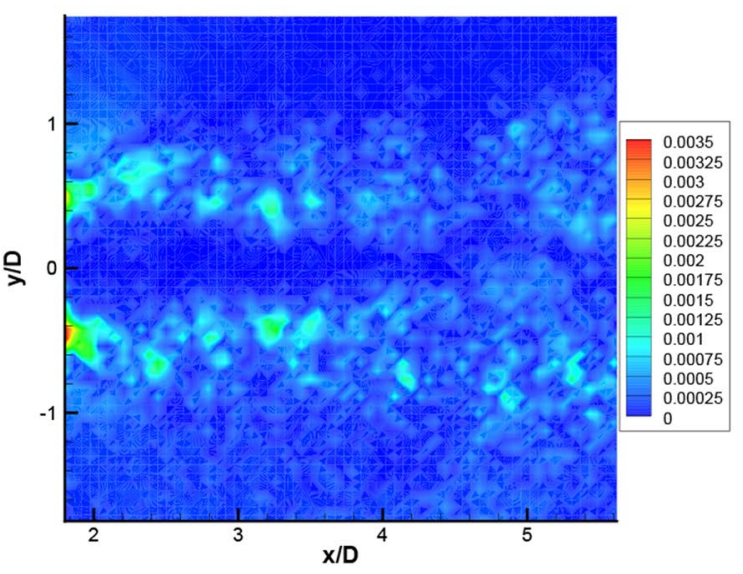

(e)

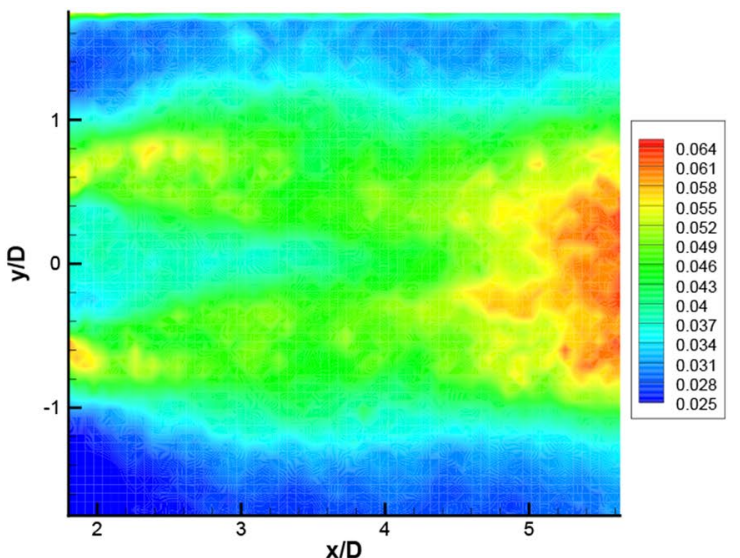

(b)

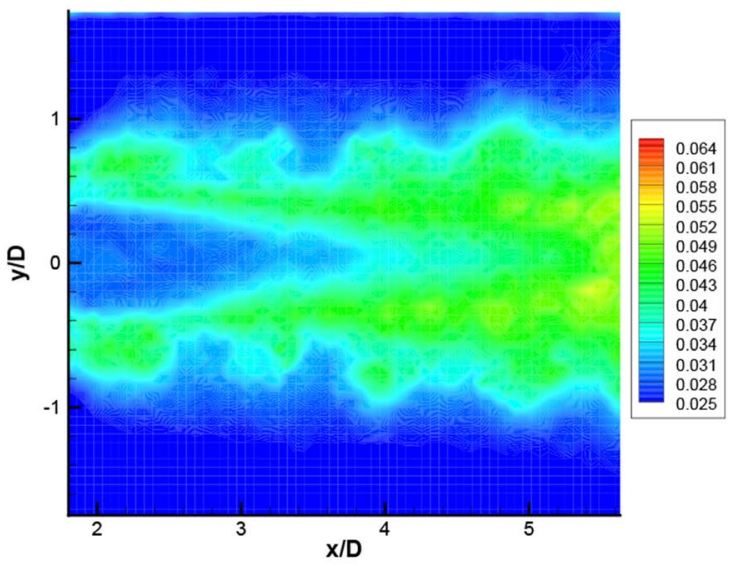

(d)

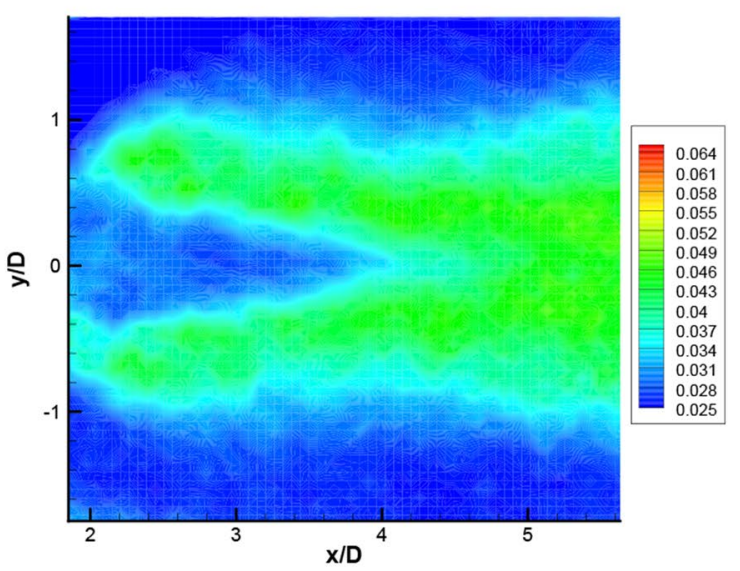

(f)

Fig. 13 Comparison between: left: phase-correlated radial fluctuation field; right: turbulent radial fluctuation field. a, b Square injection strategy; c, $\mathbf{d}$ isosceles injection strategy; e, f LAHD injection strategy. The units are $(\mathrm{m} / \mathrm{s})$

shown that the square injection strategy has the highest values of entraining inward radial velocity to which does not correspond a high mass entrainment, potentially due to its extremely abrupt deceleration. While the $L A H D$ strategy has a large inward radial velocity which does correspond to a high mass entrainment, possibly sustained by the entrainment wave triggered by the strong but not abrupt deceleration. The steep deceleration of the square 


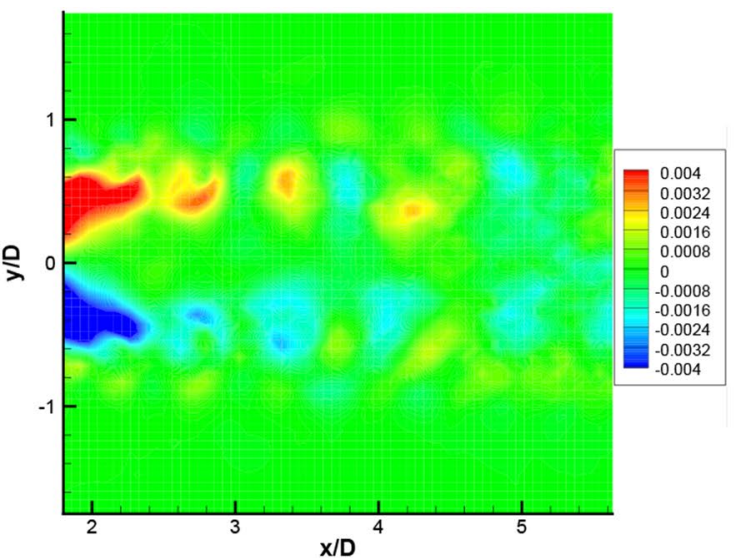

(a)

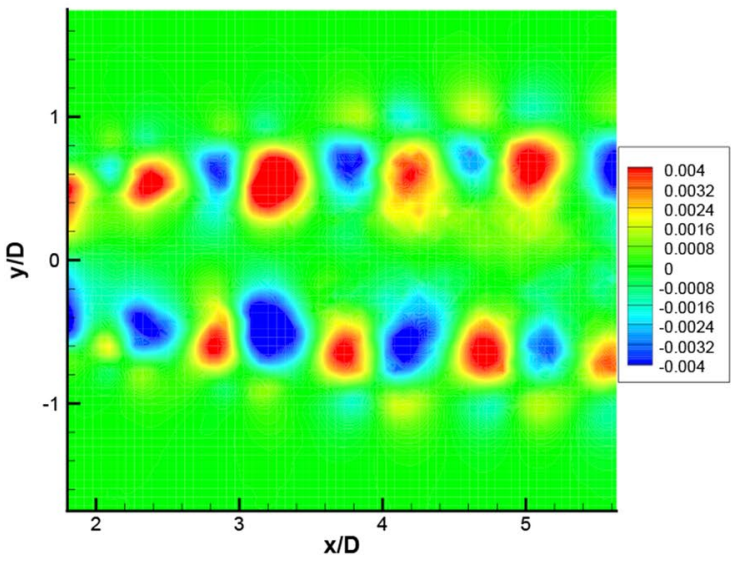

(c)

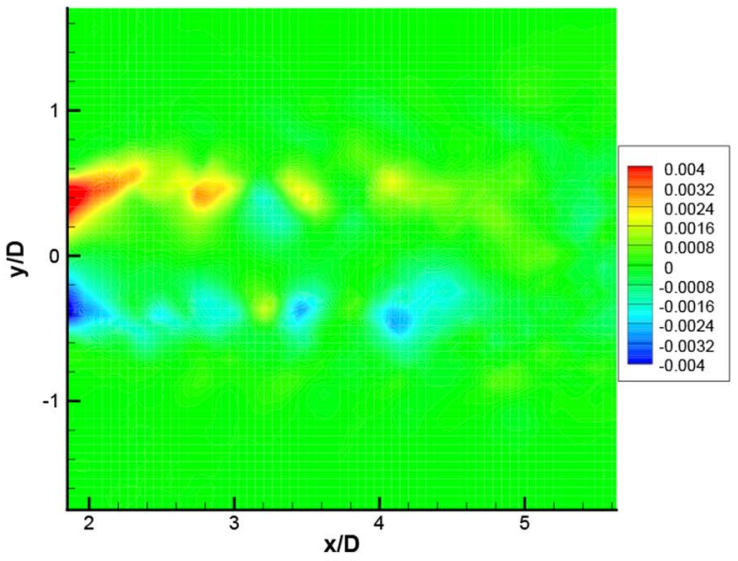

(e)

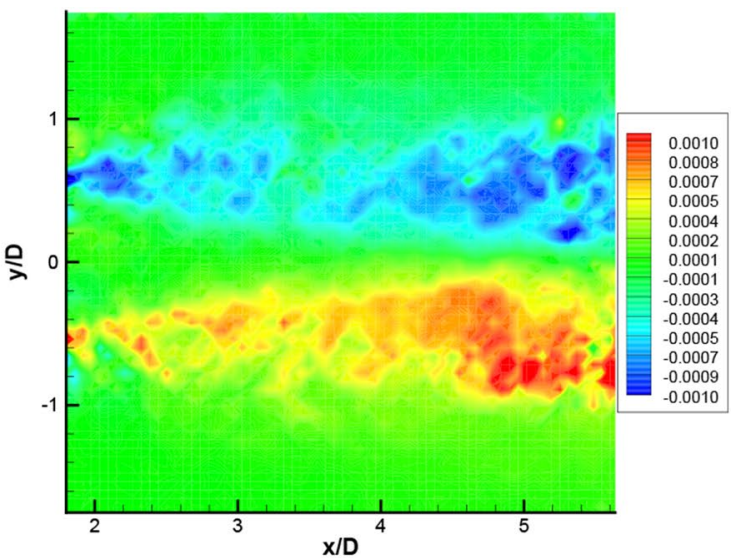

(b)

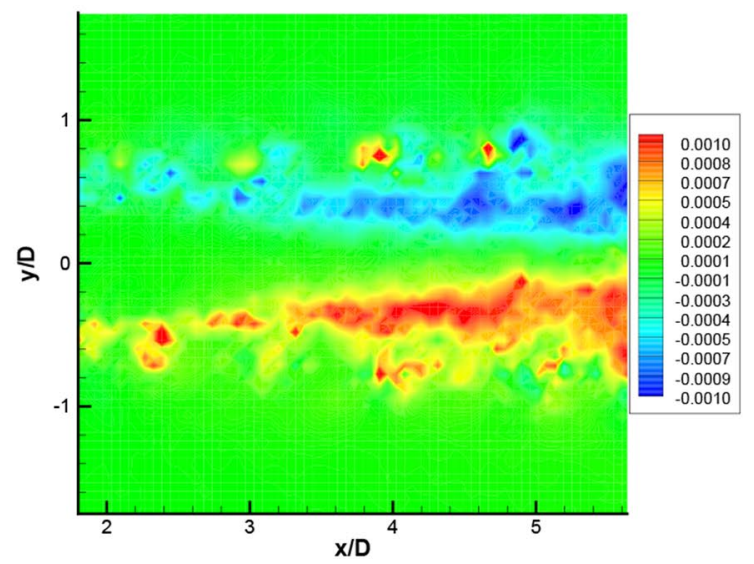

(d)

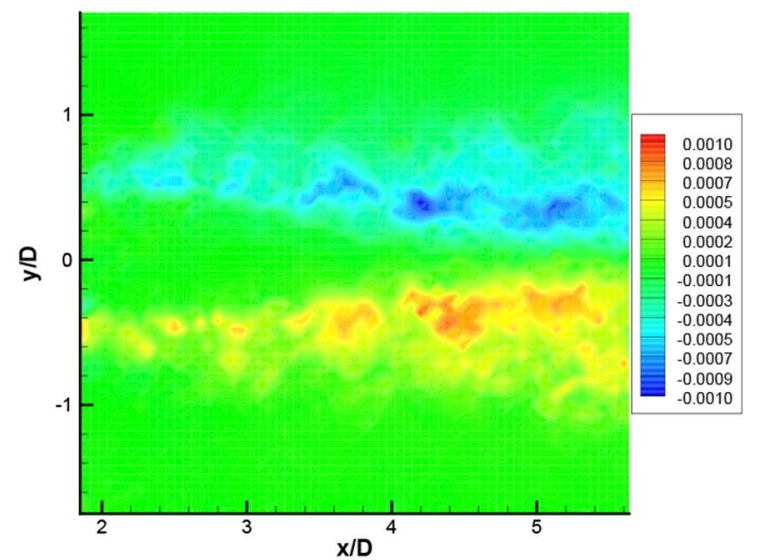

(f)

Fig. 14 Comparison between: left: phase-correlated Reynolds stress field; right: turbulent Reynolds stress field. a, b Square injection strategy; c, d isosceles injection strategy; e, f LAHD injection strategy. The units are $(\mathrm{m} / \mathrm{s})$

strategy is anyhow able to develop the highest values of turbulent mixing as reflected by the magnitude of the turbulent fluctuations. This effect increases while the vortex moves forward to the far field.
Among the square injection strategies, it has been found that a shorter injection to rest ratio $\frac{T_{1}}{T_{2}}$ is able to deliver a higher mass entrainment. Moreover, the vortex ring in these 
cases is able to travel downstream more rapidly, as predicted by the model of Musculus (2009).

The organized systematic variations in velocity, associated with the periodic flow, have been discriminated from the chaotic or turbulent ones, by means of a Triple Decomposition, and the two contributions have been contrasted for the different injection strategies.

Acknowledgements The support of this work by the Swedish Research Council (Vetenskapsrådet) through the Linné FLOW Centre is gratefully acknowledged. The help of Dr. J Töger (Lund University) with the pulse duplicator is highly appreciated.

Open Access This article is distributed under the terms of the Creative Commons Attribution 4.0 International License (http://creativeco mmons.org/licenses/by/4.0/), which permits unrestricted use, distribution, and reproduction in any medium, provided you give appropriate credit to the original author(s) and the source, provide a link to the Creative Commons license, and indicate if changes were made.

\section{References}

Adrian RJ (2005) Twenty years of particle image velocimetry. Exp Fluids 39(2):159-169

Adrian RJ, Westerweel J (2011) Particle image velocimetry, vol 30. Cambridge University Press, Cambridge

Borée J, Atassi N, Charnay G, Taubert L (1997) Measurements and image analysis of the turbulent field in an axisymmetric jet subject to a sudden velocity decrease. Exp Therm Fluid Sci 14(1):45-51

Bremhorst K, Hollis PG (1990) Velocity field of an axisymmetric pulsed, subsonic air jet. AIAA J 28(12):2043-2049

Cierpka C, Lütke B, Kähler CJ (2013) Higher order multi-frame particle tracking velocimetry. Exp Fluids 54(5):1533

Crow SC, Champagne F (1971) Orderly structure in jet turbulence. J Fluid Mech 48(3):547-591

Dabiri JO, Gharib M (2004) Fluid entrainment by isolated vortex rings. J Fluid Mech 511:311-331

Deissler RG (1958) On the decay of homogeneous turbulence before the final period. Phys Fluids 1(2):111-121

Deissler RG (1998) Turbulent fluid motion. CRC Press, Boca Raton

Doudou A (2005) Turbulent flow study of an isothermal diesel spray injected by a common rail system. Fuel 84(2):287-298

European Commission (2007) Proposal for a regulation of the European parliament and of the council setting emission performance standards for new passenger cars as part of the community's integrated approach to reduce $\mathrm{CO}_{2}$ emissions from light-duty vehicles. Tech. Rep. COM/2007/0856

European Commission (2014) Commission staff working document impact assessment accompanying the document strategy for reducing heavy-duty vehicles fuel consumption and $\mathrm{CO}_{2}$ emissions. Tech. Rep. SWD/2014/0160 final

Gharib M, Rambod E, Shariff K (1998) A universal time scale for vortex ring formation. J Fluid Mech 360:121-140

Grosshans H, Szász RZ, Fuchs L (2015) Enhanced liquid-gas mixing due to pulsating injection. Comput Fluids 107:196-204

Hu B, Musculus MP, Oefelein JC (2012) The influence of large-scale structures on entrainment in a decelerating transient turbulent jet revealed by large eddy simulation. Phys Fluids 24(4):045,106

Huang H, Fiedler H, Wang J (1993) Limitation and improvement of PIV. Exp Fluids 15(4-5):263-273

Hussain AKMF, Reynolds WC (1970) The mechanics of an organized wave in turbulent shear flow. J Fluid Mech 41(02):241-258
Jambunathan K, Ju X, Dobbins B, Ashforth-Frost S (1995) An improved cross correlation technique for particle image velocimetry. Meas Sci Technol 6(5):507

Johari H, Paduano R (1997) Dilution and mixing in an unsteady jet. Exp Fluids 23(4):272-280

Krueger PS (2001) The significance of vortex ring formation and nozzle exit over-pressure to pulsatile jet propulsion. Ph.D. thesis, Caltech

Lacagnina G, Romano G (2015) PIV investigations on optical magnification and small scales in the near-field of an orifice jet. Exp Fluids 56(1):1-11

Liepmann D, Gharib M (1992) The role of streamwise vorticity in the near-field entrainment of round jets. J Fluid Mech 245:643-668

Musculus MP (2009) Entrainment waves in decelerating transient turbulent jets. J Fluid Mech 638:117-140

Nathan G, Mi J, Alwahabi Z, Newbold G, Nobes D (2006) Impacts of a jet's exit flow pattern on mixing and combustion performance. Prog Energy Combust Sci 32(5):496-538

Nogueira J, Lecuona A, Rodriguez P (1999) Local field correction PIV: on the increase of accuracy of digital PIV systems. Exp Fluids 27(2):107-116

Olcay AB, Krueger PS (2008) Measurement of ambient fluid entrainment during laminar vortex ring formation. Exp Fluids 44(2):235-247

Querzoli G, Falchi M, Romano GP (2010) On the flow field generated by a gradually varying flow through an orifice. Eur J Mech B Fluid 29(4):259-268

Quinn W (2006) Upstream nozzle shaping effects on near field flow in round turbulent free jets. Eur J Mech B Fluids 25(3):279-301

Quinn W, Azad M, Groulx D (2012) Mean streamwise centerline velocity decay and entrainment in triangular and circular jets. AIAA J 51(1):70-79

Raffel M, Willert CE, Kompenhans J et al (2007) Particle image velocimetry: a practical guide. Springer Science \& Business Media, Berlin

Reynolds O (1895) On the dynamical theory of incompressible viscous fluids and the determination of the criterion. Philos Trans R Soc Lond A 186:123-164

Scarano F (2001) Iterative image deformation methods in PIV. Meas Sci Technol 13(1):R1

Soloff SM, Adrian RJ, Liu ZC (1997) Distortion compensation for generalized stereoscopic particle image velocimetry. Meas Sci Technol 8(12):1441

Soria J (1996) An investigation of the near wake of a circular cylinder using a video-based digital cross-correlation particle image velocimetry technique. Exp Therm Fluid Sci 12(2):221-233

Tanaka Y (1984) On the structure of pulse jet. Bull JSME 27(230):1667-1674

Töger J, Bidhult S, Revstedt J, Carlsson M, Arheden H, Heiberg E (2015) Independent validation of four-dimensional flow MR velocities and vortex ring volume using particle imaging velocimetry and planar laser-induced fluorescence. Magn Reson Med 75(3): 1064-1075

Westerweel J, Scarano F (2005) Universal outlier detection for PIV data. Exp Fluids 39(6):1096-1100

Willert CE (2006) Assessment of camera models for use in planar velocimetry calibration. Exp Fluids 41(1):135-143

Willert CE, Gharib M (1991) Digital particle image velocimetry. Exp Fluids 10(4):181-193

Wygnanski I, Fiedler H (1969) Some measurements in the self-preserving jet. J Fluid Mech 38(3):577-612

Publisher's Note Springer Nature remains neutral with regard to jurisdictional claims in published maps and institutional affiliations. 